\title{
LIFTING PRIMITIVITY OF FREE NILPOTENT GROUPS
}

\author{
C. K. GUPTA AND N. D. GUPTA \\ (Communicated by Warren J. Wong) \\ Dedicated to Horace Mochizuki
}

\begin{abstract}
Let $F_{n, c}$ denote the free $n$-generator nilpotent group of class $c$. For $n \geq c+m-1$, every primitive system of $m$ elements of $F_{n, c}$ can be lifted to a primitive system of $m$ elements of the free group $F_{n}$.
\end{abstract}

\section{INTRODUCTION}

Let $F=F_{n}, n \geq 2$, be a free group and $V$ be a fully invariant subgroup of $F$. A system $\mathbf{w}=\left(w_{1}, \ldots, w_{m}\right), m \leq n, w_{i} \in F$, is said to be primitive in $F$ if $\mathbf{w}$ can be extended to a basis of $F$, and $\mathbf{w}$ is said to be primitive $\bmod V$ if the corresponding system $\overline{\mathbf{w}}=\left(\bar{w}_{1}, \ldots, \bar{w}_{m}\right)$ of cosets $\bar{w}_{i}\left(=w_{i} V\right)$ can be extended to some basis of $F / V$. If $\mathbf{w}=\left(w_{1}, \ldots, w_{m}\right)$ is primitive $\bmod V$ and if there exists $\mathbf{v}=\left(v_{1}, \ldots, v_{m}\right), v_{i} \in V$, such that the corresponding system $\mathbf{w}^{*}=\left(w_{1} v_{1}, \ldots, w_{m} v_{m}\right)$ is primitive in $F$ then we say that $\mathbf{w}$ lifts to $\mathbf{w}^{*}$. Lifting primitivity of free $V$-groups is a natural problem which is linked to the study of the automorphism groups $\operatorname{Aut}(F / V)$. For $n \geq 4, m \leq n$, every primitive system $w \bmod F^{\prime \prime}$ lifts to a primitive system $\mathbf{w}^{*}$ of $F$. This follows from the well-known result of Bachmuth and Mochizuki [3] that every automorphism of a free metabelian group of rank exceeding 3 is tame (i.e., induced by an automorphism of $F$ ). When $F / V$ is free nilpotent of class exceeding two or free metabelian nilpotent of class exceeding two then there exist nontame automorphisms for arbitrary large values of $n[1,2,4]$. In [5] we studied the lifting of primitivity of free metabelian nilpotent groups and proved that for $n \geq m+2$, every primitive system $w \bmod \gamma_{c+1}(F) F^{\prime \prime}$ lifts to a primitive system $\mathbf{w}^{*}$ of $F$. In this paper we study the corresponding problem of lifting primitivity of systems of elements in free nilpotent groups $F_{n, c}\left(\cong F / \gamma_{c+1}(F)\right)$, $c \geq 2$. We prove that, for $n \geq c+m-1$, every primitive system $\mathbf{w} \bmod \gamma_{c+1}(F)$ of $m$ elements lifts to a primitive system $\mathbf{w}^{*}$ of $F$ (Theorem C).

Received by the editors September 7, 1990.

1980 Mathematics Subject Classification (1985 Revision). Primary 20F28, $20 \mathrm{~F} 18$.

Key words and phrases. Free groups, free nilpotent groups, primitive systems, automorphism groups. 


\section{PRELIMINARY LEMMAS}

We use standard commutator notation: $[a, b]=a^{-1} b^{-1} a b,[a, b, c]=$ [[a,b],c], etc. Let $F=\left\langle x_{1}, x_{2}, \ldots, x_{n}\right\rangle$ be free of rank $n$. We first prove some lemmas concerning the existence of certain specific automorphisms of $F$.

Lemma 1. For any permutation $\pi$ of the set $\{2, \ldots, c\}, 2 \leq c \leq n$, there is an automorphism $\theta_{\pi}$ of $F$ of the form given

$$
\begin{array}{r}
\theta_{\pi}=\left\{x_{1} \rightarrow x_{1}\left[x_{1}, x_{2 \pi}, \ldots, x_{c \pi}\right] v_{1}, x_{i} \rightarrow x_{i} u_{i},\right. \\
\left.i=2, \ldots, c, x_{j} \rightarrow x_{j}, j \geq c+1\right\},
\end{array}
$$

where $v_{1} \in \gamma_{c+1}(F)$ and $u_{i} \in F^{\prime}\left(=\gamma_{2}(F)\right)$.

Proof. For $c=2, \theta=\theta_{\pi}=\left\{x_{1} \rightarrow x_{1}\left[x_{1}, x_{2}\right], x_{i} \rightarrow x_{i}, i \neq 1\right\}$ is an automorphism of $F$ of the required form. For $c \geq 3$, consider endomorphisms $\alpha, \beta$ of $F$ given

$$
\begin{aligned}
& \alpha=\left\{x_{1} \rightarrow x_{1}\left[x_{2}, \ldots, x_{c}\right], x_{i} \rightarrow x_{i}, i \neq 1\right\}, \\
& \beta=\left\{x_{2} \rightarrow x_{2}\left[x_{2}, x_{1}\right], x_{i} \rightarrow x_{i}, i \neq 2\right\} .
\end{aligned}
$$

Then clearly $\alpha, \beta$ are IA-automorphisms of $F$ (i.e., inducing identity modulo $\left.F^{\prime}\right)$. Set $\theta=\alpha \beta \alpha^{-1} \beta^{-1}$. So,

and

$$
\theta\left(x_{2}\right) \equiv x_{2}\left(\bmod F^{\prime}\right), \quad \theta\left(x_{i}\right)=x_{i} \quad \text { for } i=3, \ldots, n,
$$

$\theta\left(x_{1}\right) \equiv x_{1}\left[x_{1}, x_{2}, \ldots, x_{c}\right]\left(\bmod \gamma_{c+1}(F)\right)$

(since $\beta^{-1}\left(x_{1}\right)=x_{1} ; \quad \alpha^{-1} \beta^{-1}\left(x_{1}\right)=x_{1}\left[x_{2}, \ldots, x_{c}\right]^{-1} ; \beta \alpha^{-1} \beta^{-1}\left(x_{1}\right)$ $=x_{1}\left[x_{2}\left[x_{2}, x_{1}\right], x_{3}, \ldots, x_{c}\right]^{-1} \equiv x_{1}\left[x_{2}, \ldots, x_{c}\right]^{-1}\left[x_{1}, x_{2}, x_{3}, \ldots, x_{c}\right]$ $\left.\left(\bmod \gamma_{c+1}(F)\right) ; \alpha \beta \alpha^{-1} \beta^{-1}\left(x_{1}\right) \equiv x_{1}\left[x_{1}, x_{2}, x_{3}, \ldots, x_{c}\right]\left(\bmod \gamma_{c+1}(F)\right)\right)$. For any permutation $\pi$ of the set $\{2, \ldots, c\}$ define an automorphism $\theta_{\pi}$ of $F$ by

$$
\theta_{\pi}=\pi^{*} \theta \pi^{*-1}
$$

where $\theta=\alpha \beta \alpha^{-1} \beta^{-1}$ is as above and $\pi^{*}$ is given by

$$
\pi^{*}=\left\{x_{1} \rightarrow x_{1}, x_{2} \rightarrow x_{2 \pi}, \ldots, x_{c} \rightarrow x_{c \pi}, x_{j} \rightarrow x_{j}, j \geq c+1\right\} .
$$

Then it is clear that $\theta_{\pi}$ is of the required form:

$$
\begin{array}{r}
\theta_{\pi}=\left\{x_{1} \rightarrow x_{1}\left[x_{1}, x_{2 \pi}, \ldots, x_{c \pi}\right] v_{1}, x_{i} \rightarrow x_{i} u_{i},\right. \\
\left.i=2, \ldots, c, x_{j} \rightarrow x_{j}, j \geq c+1\right\},
\end{array}
$$

where $v_{1} \in \gamma_{c+1}(F)$ and $u_{i} \in F^{\prime}$.

Lemma 2. Given $\left[x_{1}, y_{1}, \ldots, y_{c-1}\right], 2 \leq c \leq n, y_{i} \in\left\{x_{1}, x_{2}, \ldots, x_{n}\right\} \quad\left(y_{1} \neq\right.$ $\left.x_{1}\right)$, there exists $\mu \in \operatorname{Aut}(F)$ such that $\mu\left(x_{1}\right) \equiv x_{1}\left[x_{1}, y_{1}, \ldots, y_{c-1}\right]$ $\left(\bmod \gamma_{c+1}(F)\right) ; \mu\left(x_{i}\right) \equiv x_{i}\left(F^{\prime}\right), 2 \leq i \leq c$, and $\mu\left(x_{j}\right)=x_{j}, j \geq c+1$.

Proof. Let

$$
\begin{aligned}
\mathbf{z}(k)=\left(z_{1}, z_{2}, \ldots, z_{k}\right) & =\left(x_{i 1}, \ldots, x_{i k}\right), \\
2 & \leq i 1<\cdots<i k \leq c, 1 \leq k \leq c-1,
\end{aligned}
$$

be an arbitrary $k$-tuple of distinct generators of $F$ and let

$$
\mathbf{y}(k)=\left(y_{1}, y_{2}, \ldots, y_{k}\right), y_{i} \in\left\{x_{1}, \ldots, x_{n}\right\}, 1 \leq k \leq c-1,
$$

be an arbitrary $k$-tuple. 
Let $u(\mathbf{z}(k) \rightarrow \mathbf{y}(k))$ denote the commutator obtained from $u=\left[x_{1}, x_{2}, x_{3}\right.$, $\ldots, x_{c}$ ] upon replacing the $k$-tuple $\mathbf{z}(k)$ by the $k$-tuple $\mathbf{y}(k)$. We prove by induction on $1 \leq k \leq c-1$ that there is an automorphism $\mu(\mathbf{z}(k) \rightarrow \mathbf{y}(k))$ of $F$ such that

$\mu(\mathbf{z}(k) \rightarrow \mathbf{y}(k)) x_{1} \equiv x_{1} u(\mathbf{z}(k) \rightarrow \mathbf{y}(k))\left(\bmod \gamma_{c+1}(F)\right) ; \mu(\mathbf{z}(k) \rightarrow \mathbf{y}(k))\left(x_{k}\right) \equiv x_{i}$

$\left(\bmod F^{\prime}\right), 2 \leq i \leq c$, and $\mu(\mathbf{z}(k) \rightarrow \mathbf{y}(k)) \quad\left(x_{j}\right)=x_{j}, j \geq c+1$. The statement of the lemma is the case $k=c-1$.

For $k=1$, if $z_{1}=y_{1}$ then there is nothing to prove. Otherwise, we consider

$$
\alpha=\left\{z_{1} \rightarrow z_{1} y_{1}, x_{j} \rightarrow x_{j}, x_{j} \neq z_{1}\right\} \in \operatorname{Aut}(F)
$$

and, by Lemma 1, the automorphism $\theta$ of $F$ given by

$$
\begin{array}{r}
\theta=\left\{x_{1} \rightarrow x_{1}\left[x_{1}, x_{2}, \ldots, x_{c}\right] v_{1}, x_{i} \rightarrow x_{i} u_{i},\right. \\
\\
\left.i=2, \ldots, c, x_{j} \rightarrow x_{j}, j \geq c+1\right\},
\end{array}
$$

where $v_{1} \in \gamma_{c+1}(F), u_{i} \in F^{\prime}$. We define

$$
\mu(\mathbf{z}(1) \rightarrow \mathbf{y}(1))=\alpha^{-1} \theta \alpha \theta^{-1}
$$

and verify instantly that it is of the required form.

For $k=2, \ldots, c-1$, assume the result for $k-1$.

Consider $\mathbf{y}(k)=\left(y_{1}, y_{2}, \ldots, y_{k}\right)$ and define for each $t=1, \ldots, k$,

$$
\mathbf{y}(k ; t)=\left(y_{1}, \ldots, y_{t-1}, z_{t}, y_{t+1}, \ldots, y_{k}\right) \text {. }
$$

Then by the induction hypothesis there exist automorphisms $\mu(\mathbf{z}(k), \mathbf{y}(k ; t))$, $t=1, \ldots, k$, such that $\mu(\mathbf{z}(k) \rightarrow \mathbf{y}(k ; t)) \quad\left(x_{1}\right) \equiv x_{1} u(\mathbf{z}(k) \rightarrow \mathbf{y}(k ; t))$ $\left(\bmod \gamma_{c+1}(F)\right) ; \mu(\mathbf{z}(k) \rightarrow \mathbf{y}(k ; t))\left(x_{i}\right) \equiv x_{i}\left(\bmod F^{\prime}\right), 2 \leq i \leq c$, and $\mu(\mathbf{z}(k) \rightarrow \mathbf{y}(k ; t)) \quad\left(x_{j}\right)=x_{j}, j \geq c+1$.

In particular, if $z_{t}=y_{t}$ for some $t=1, \ldots, k$ then, for that value of $t, \mu(\mathbf{z}(k) \rightarrow \mathbf{y}(k))=\mu(\mathbf{z}(k) \rightarrow \mathbf{y}(k ; t))$ is an automorphism of the required form. Thus we may assume that $z_{t} \neq y_{t}$ for all $t=1, \ldots, k$. Further, if $z_{t} \in\left\{y_{1}, \ldots, y_{t-1}, y_{t+1}, \ldots, y_{k}\right\}$ for each $t=1, \ldots, k$, then $\mathbf{y}(k)$ is a permutation of $\mathbf{z}(k)$ and by Lemma 1 there exists an automorphism of the required form. Thus we may assume, without loss of generality, that $z_{t} \notin$ $\left\{y_{1}, \ldots, y_{k}\right\}$ for some $1 \leq t \leq k$. Define

$$
\mu(\mathbf{z}(k) \rightarrow \mathbf{y}(k))=\alpha_{t}^{-1} \mu(\mathbf{z}(k) \rightarrow \mathbf{y}(k ; t)) \alpha_{t} \mu(\mathbf{z}(k) \rightarrow \mathbf{y}(k ; t))^{-1},
$$

where $\alpha_{t}=\left\{z_{t} \rightarrow z_{t} y_{t}, x_{i} \rightarrow x_{i}, x_{i} \neq z_{t}\right\}$.

It follows immediately that $\mu(\mathbf{z}(k) \rightarrow \mathbf{y}(k))$ is an automorphism of $F$ which has the required properties.

As a corollary to Lemma 2 we deduce,

Lemma 3. Let $u=u^{\prime}\left(\hat{x}_{1}\right) \prod_{\mathbf{y}}\left[x_{1}, y_{1}, \ldots, y_{c-1}\right]^{a(\mathbf{y})}, c \geq 2$, be an element of $\gamma_{c}(F)$ where the component $u^{\prime}\left(\hat{x}_{1}\right)$ does not involve the generator $x_{1}$ and $\mathbf{y}=$ $\left(y_{2}, \ldots, y_{c-1}\right)$ with $y_{i} \in\left\{x_{1}, x_{2}, \ldots, x_{n}\right\} \quad\left(y_{1} \neq x_{1}\right), a(\mathbf{y}) \in \mathbf{Z}$. Then there is an IA-automorphism $\mu$ of $F$ such that $\mu\left(x_{1}\right) \equiv x_{1} u\left(\bmod \gamma_{c+1}(F)\right), \mu\left(x_{i}\right) \equiv x_{i}$ $\left(\bmod F^{\prime}\right)$ for $2 \leq i \leq c$, and $\mu\left(x_{j}\right)=x_{j}$ for $j \geq c+1$.

Proof. Since $u^{\prime}\left(\hat{x}_{1}\right)$ does not involve the generator $x_{1}$, there is an automorphism $\alpha$ of $F$ of the form given by $\alpha=\left\{x_{1} \rightarrow x_{1} u^{\prime}\left(\hat{x}_{1}\right), x_{i} \rightarrow x_{i}, i \neq 1\right\}$. 
Thus applying $\alpha^{-1}$ to $u$, we may assume $u=\prod_{\mathrm{y}}\left[x_{1}, y_{1}, \ldots, y_{c-1}\right]^{a(\mathrm{y})}$. By Lemma 2, for each $\left[x_{1}, y_{1}, \ldots, y_{c-1}\right]$ there is an IA-automorphism $\mu(\mathbf{z} \rightarrow \mathbf{y}) \in$ $\operatorname{Aut}(F)$ such that $\mu(\mathbf{z} \rightarrow \mathbf{y})\left(x_{1}\right) \equiv x_{1}\left[x_{1}, y_{1}, \ldots, y_{c}\right]\left(\bmod \gamma_{c+1}(F)\right), \mu(\mathbf{z} \rightarrow \mathbf{y})$ $\left(x_{i}\right) \equiv x_{i}\left(\bmod F^{\prime}\right)$ for $2 \leq i \leq c$, and $\mu(\mathbf{z} \rightarrow \mathbf{y})\left(x_{j}\right)=x_{j}$ for $j \geq c+1$. Define $\mu=\prod_{y}(\mu(\mathbf{z} \rightarrow \mathbf{y}))^{a(\mathbf{y})}$. It is easily verified that $\mu$ has the required properties.

\section{LIFTING PRIMITIVITY OF SYSTEMS OF ELEMENT}

We first establish the lifting of a single primitive element. We first prove,

Theorem A. Let $n \geq c \geq 2$ and $u \in F^{\prime}$ be fixed. Then there is an IAautomorphism $\delta$ of $F$ such that $\delta\left(x_{1} u\right) \equiv x_{1}\left(\bmod \gamma_{c+1}(F)\right), \delta\left(x_{i}\right) \equiv x_{i}$ $\left(\bmod F^{\prime}\right)$ for $i=2, \ldots, c$, and $\delta\left(x_{j}\right)=x_{j}$ for $j=c+1, \ldots, n$.

Proof. Repeated applications of Lemma 3 yield IA-automorphisms $\delta_{2}, \delta_{3}, \ldots$, $\delta_{c}$ of $F$ satisfying,

$$
\begin{aligned}
& \delta_{2}\left(x_{1} u\right)=x_{1} u_{3}, \quad u_{3} \in \gamma_{3}(F) \quad \text { and } \quad \delta_{2}\left(x_{j}\right)=x_{j} \quad \text { for } j \geq 3 . \\
& \delta_{3}\left(x_{1} u_{3}\right)=x_{1} u_{4}, \quad u_{4} \in \gamma_{4}(F) \quad \text { and } \quad \delta_{3}\left(x_{j}\right)=x_{j} \text { for } j \geq 4 ; \\
& \delta_{c}\left(x_{1} u_{c}\right)=x_{1} u_{c+1}, \quad u_{c+1} \in \gamma_{c+1}^{\prime}(F) \quad \text { and } \quad \delta_{c}\left(x_{j}\right)=x_{j}=x_{j} \quad \text { for } j \geq c+1 .
\end{aligned}
$$

Thus $\delta=\delta_{c} \cdots \delta_{3} \delta_{2}$ is such that $\delta\left(x_{1} u\right) \equiv x_{1}\left(\bmod \gamma_{c+1}(F)\right), \delta\left(x_{i}\right) \equiv x_{i}$ $\left(\bmod F^{\prime}\right)$ for $i=2, \ldots, c$, and $\delta\left(x_{j}\right)=x_{j}$ for $j \geq c+1$, as required.

We can now prove primitive lifting of a single element.

Theorem B. Let $n \geq c \geq 2$ be fixed and let $w \in F$ be primitive $\bmod \gamma_{c+1}(F)$. Then $w$ can be lifted to a primitive element of $F$.

Proof. Let $w \in F$ be primitive $\bmod \gamma_{c+1}(F)$. For any automorphism $\xi$ of $F$, the set $\left\{\xi\left(x_{1}\right), \xi\left(x_{2}\right), \ldots, \xi\left(x_{n}\right)\right\}$ forms a basis of $F$ and it suffices, therefore, to prove that there exists $\xi \in \operatorname{Aut}(F)$ with $\xi\left(x_{1}\right)=w v$ for some $v$ in $\gamma_{c+1}(F)$. Since $w$ is primitive $\bmod F^{\prime}$, there exists an automorphism $\alpha \in \operatorname{Aut}(F)$ such that $\alpha(w)=x_{1} u$ for some $u$ in $F^{\prime}$. By Theorem A, there exists an automorphism $\delta$ of $F$ such that $\delta^{-1}\left(x_{1}\right) \equiv x_{1} u\left(\bmod \gamma_{c+1}(F)\right)$, so that $\alpha^{-1} \delta^{-1}\left(x_{1}\right) \equiv w \bmod \gamma_{c+1}(F)$. Thus $\xi=\alpha^{-1} \delta^{-1}$ is an automorphism of $F$ with $\xi\left(x_{1}\right)=w v$ for some $v$ in $\gamma_{c+1}$. This completes the proof of the theorem.

Let $\mathbf{w}=\left(w_{1}, \ldots, w_{m}\right), m \geq 1$, be an arbitrary system of primitive elements $\bmod \gamma_{c+1}(F)$. We can now prove our principal result of the paper.

Theorem C. For $n \geq c+m-1$, every primitive system

$$
\mathbf{w}=\left(w_{1}, \ldots, w_{m}\right) \bmod \gamma_{c+1}(F)
$$

can be lifted to a primitive system of $F$.

Proof. As before we may assume, without loss of generality, that $\mathbf{w}$ is of the form $\mathbf{w}=\left(x_{1} u_{1}, \ldots, x_{m} u_{m}\right), u_{i} \in F^{\prime}$. So the problem reduces to showing that there exist $v_{i} \in \gamma_{c+1}(F), i=1, \ldots, m$, such that

$$
\mathbf{w}^{*}=\left(x_{1} u_{1} v_{1}, \ldots, x_{m} u_{m} v_{m}\right)
$$

is a primitive system of $F$. We achieve this by proving, by induction on $m \geq 1$, that for the given system $\mathbf{w}=\left(x_{1} u_{1}, \ldots, x_{m} u_{m}\right)$ there is an IAautomorphism $\sigma(\mathbf{w})$ of $F$ such that $\sigma(\mathbf{w})\left(x_{i}\right) \equiv x_{i} u_{i}\left(\bmod \gamma_{c+1}(F)\right)$ for $i=$ 
$1, \ldots, m$. For $m=1$, the result comes from Theorem A. Let $m \geq 2$ and assume the result for $m-1$. By the induction hypothesis, the subsystem $\left(x_{2} u_{2}, \ldots, x_{m} u_{m}\right)$ lifts to a primitive subsystem $\left(x_{2} u_{2} v_{2}, \ldots, x_{m} u_{m} v_{m}\right)$ of $F$. So there is an IA-automorphism $\xi$ such that $\xi\left(x_{i} u_{i}\right) \equiv x_{i}\left(\bmod \gamma_{c+1}(F)\right)$, $i=2, \ldots, m$. Applying $\xi$ to the given system $\mathbf{w}=\left(x_{1} u_{1}, \ldots, x_{m} u_{m}\right)$ shows that $\xi(\mathbf{w})=\left(x_{1} u_{1}^{*}, x_{2} v_{2}, \ldots, x_{m} v_{m}\right)$ where $u_{1}^{*} \in F^{\prime}, v_{i} \in \gamma_{c+1}(F)$. Thus it suffices to exhibit an automorphism $\delta$ of $F$ with the property that $\delta\left(x_{1} u_{1}^{*}\right) \equiv$ $x_{1}\left(\bmod \gamma_{c+1}(F)\right)$ and $\delta\left(x_{i}\right)=x_{i}$ for $i=2, \ldots, m$. Indeed, since $n \geq$ $c+m-1$, Theorem A yields an automorphism $\delta$ such that $\delta\left(x_{1} u_{1}^{*}\right) \equiv x_{1}$ $\left(\bmod \gamma_{c+1}(F)\right), \delta\left(x_{i}\right) \equiv x_{i}\left(\bmod F^{\prime}\right)$ for $i=m+1, \ldots, c+m-1$, and $\delta\left(x_{j}\right)=x_{j}$ for $j=2, \ldots, m, c+m, \ldots, n$. This completes the proof of the theorem.

\section{CONCLUDING REMARKS}

For $n=2, c \geq 3$, there is a primitive element $\bmod \gamma_{c+1}(F)$ which cannot be lifted to a primitive element of $F$, and for $n=3, c \geq 3$, there is a primitive system of two elements $\bmod \gamma_{c+1}(F)$ which cannot be lifted to a primitive system of $F$ (see [5, Remarks]). For $c \geq 4, n=c-1$, it would be of interest to know whether every primitive element $\bmod \gamma_{c+1}(F)$ can be lifted to a primitive element of $F$. The simplest case of the problem is to decide whether or not, for $n=3, c=4$, the element $x_{1}\left[x_{1}, x_{2}, x_{2}, x_{3}\right]$ can be lifted to a basis of $F$.

Added in proof. A weaker form of Theorem $\mathrm{C}$ is also implicit in Bryant and Macedonska [J. Algebra 121 (1989), 388-398] where it is shown that for sufficiently large $n=n(m, c)$ every primitive system $\bmod \gamma_{c+1}\left(F_{n}\right)$ can be lifted to a primitive system of $F_{n}$.

\section{REFERENCES}

1. S. Andreadakis, On the automorphisms of free groups and free nilpotent groups, Proc. London Math. Soc. (3) 15 (1965), 239-268.

2. S. Bachmuth, Induced automorphisms of free groups and free metabelian groups, Trans. Amer. Math. Soc. 122 (1966), 1-17.

3. S. Bachmuth and $\mathrm{H}$. Y. Mochizuki, $\operatorname{Aut}(F) \rightarrow \operatorname{Aut}\left(F / F^{\prime \prime}\right)$ is surjective for free group $F$ of rank $\geq 4$, Trans. Amer. Math. Soc. 292 (1985), 81-101.

4. R. M. Bryant, C. K. Gupta, F. Levin, and H. Y. Mochizuki, Non-tame automorphisms of free nilpotent groups, Comm. Algebra 18 (1990), 3619-3631.

5. C. K. Gupta, N. D. Gupta, and V. A. Romankov, Primitivity in free groups and free metabelian groups, Canad. J. Math. (to appear).

Department of Mathematics, University of Manitoba, Winnipeg R3T 2N2, Canada 